\title{
6th Grade Completion
}

National Cancer Institute

\section{Source}

National Cancer Institute. 6th Grade Completion. NCI Thesaurus. Code C67128.

Indicates that 6 th grade is the highest level of educational achievement. 